\title{
Ruptured intracranial aneurysms: has the incidence of early rebleeding been over-estimated?
}

\author{
RS MAURICE-WILLIAMS \\ From the Royal Free Hospital, London, UK
}

SUMMARY Of 110 consecutive patients with ruptured intracranial aneurysms, 49 underwent delayed neurological deterioration, involving 57 episodes in all. During the first three weeks after the presenting haemorrhage less than a third of these episodes were due to confirmed rebleeding. Rebleeding episodes were found to have a "flat" distribution in time during this period, but episodes of non-haemorrhagic deterioration "peaked" between days 4-12. This peak coincides with the peak for rebleeding which was described in earlier studies on aneurysms, and it is suggested that confusion between non-haemorrhagic deterioration and rebleeding may have led to a significant over-estimate of the incidence of early rebleeding with important implications for the optimum timing of surgical intervention.

Many of the patients who reach hospital in a good condition after an aneurysmal subarachnoid haemorrhage and who deteriorate over the next few weeks do so from causes other than recurrent bleeding. The pioneer studies of the natural history of intracranial aneurysms, which are still generally used as the basis for decisions about surgical treatment, paid little or no attention to this fact. As a consequence they may have seriously overestimated the incidence of early recurrent haemorrhage. Detailed observations carried out on a series of 110 consecutive ruptured aneurysms suggest that this is indeed the case. This could have important consequences both for the surgical management of aneurysms and for the interpretation of studies which show apparent benefit from antifibrinolytic treatment.

\section{Material}

One hundred and ten consecutive patients with a confirmed diagnosis of ruptured intracranial aneurysm admitted under the care of a single consultant neurosurgeon were studied. During the period of study every patient under the age of 65 years referred with a diagnosis of subarachnoid haemorrhage was admitted within 12 hours of referral regardless of neurological condition. Seventy-seven patients $(70 \%)$ were admitted within 72 hours of the haemorrhage. Clinical grade on admission is shown in table 1 .

Address for reprint requests: Mr RS Maurice-Williams, The Royal Free Hospital, Pond St, London NW3 2QG, UK

Received 6 March 1982 and in revised form 27 April 1982. Accepted 5 May 1982
Table 1 Relationship between clinical grade on admission, ${ }^{*}$ confirmed rebleeding and non-haemorrhagic deterioration (NHD)

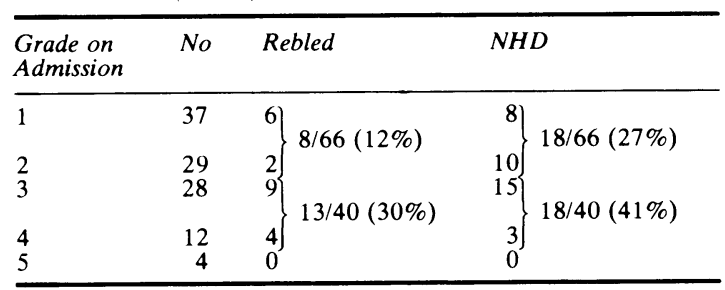

${ }^{*}$ Botterell Grades as modified by Hunt and Hess. ${ }^{3}$

On admission CT-scanning of the head was carried out and antifibrinolytic treatment with tranexamic acid $6 \mathrm{~g} /$ day was started. The latter was continued until operation, or for $\mathbf{4 2}$ days if no surgery was carried out. Cerebral angiography was generally performed within 3 days of admission. Surgery was not carried out within 7 days of the last haemorrhage unless the patient's life was threatened by an expanding intracranial haematoma. Even after 7 days had elapsed, surgery was postponed until the patient was both in a good neurological condition and stable or improving. Surviving patients have been followed up for between 6 months and 4 years. Seventy-one patients underwent surgery (69 intracranial operations, two common carotid ligations.) Only 23 patients underwent surgery within 10 days of the presenting haemorrhage. Ninety-seven patients $(88 \%)$ received antifibrinolytic treatment.

Patients who showed definite and sustained clinical deterioration after admission were subjected to repeat CT-scanning and lumbar puncture (and necropsy if they died) in an attempt to establish whether the deterioration was due to rebleeding. Those patients in whom no evidence of rebleeding could be found were designated as having undergone "non-haemorrhagic deterioration". 


\section{Results}

(A) REBLEEDING

(i) Incidence: 21 patients had episodes of rebleeding confirmed by lumbar puncture, CT scan or necropsy. Of these, 13 occurred within 18 days: their distribution in time is shown in figure 1. (2) Mortality: This was $90 \%(19 / 21)$, or $85 \%(11 / 13)$ of the rebleeds which occurred within the first 18 days. (3) Relationship to clinical grade on admission, age and site of aneurysm: These are shown in tables 1-3.

Table 2 Incidence of rebleeding and NHD according to site of aneurysm

\begin{tabular}{lrrl}
\hline Aneurysm site $^{*}$ & No & NHD & Rebleed \\
\hline Internal carotid & 38 & 12 & 7 \\
Anterior communicating & 28 & 14 & 6 \\
Middle cerebral & 32 & 8 & 5 \\
Distal anterior cerebral & 2 & 1 & $\frac{1}{3}$ \\
Vertebro-basilar & 10 & 1 & (10 \\
\hline
\end{tabular}

*Where a patient had multiple aneurysms, the aneurysm which had bled is counted.

Table 3 Incidence of rebleeding and NHD according to age of patient

\begin{tabular}{lccl}
\hline Age $(y r)$ & No & NHD & Rebleed \\
\hline 30 or less & 12 & 3 & 1 \\
$31-40$ & 22 & 9 & 6 \\
$41-50$ & 26 & 10 & 5 \\
$51-60$ & 43 & 13 & 8 \\
$61-65$ & 7 & 1 & 1 \\
\hline
\end{tabular}

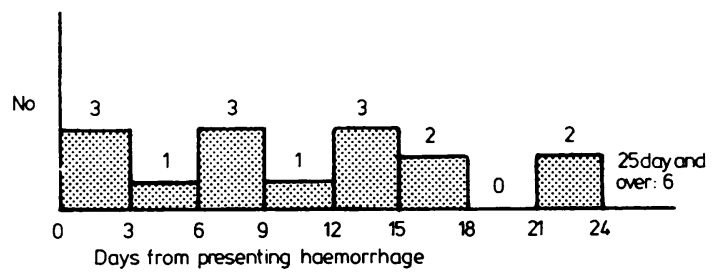

Fig 1 Time course of rebleeding.

(B) NON-HAEMORRHAGIC DETERIORATION

(1) Incidence: 36 patients showed definite clinical deterioration after admission without any evidence of recurrent bleeding. (2) Distribution in time: This is shown in fig 2. Thirty-five of the 36 episodes began within 12 days of the presenting haemorrhage. The latest episode began on Day 16. (3) Sequelae: Eight patients (22\%) died from worsening non-haemorrhagic deterioration. Five died from

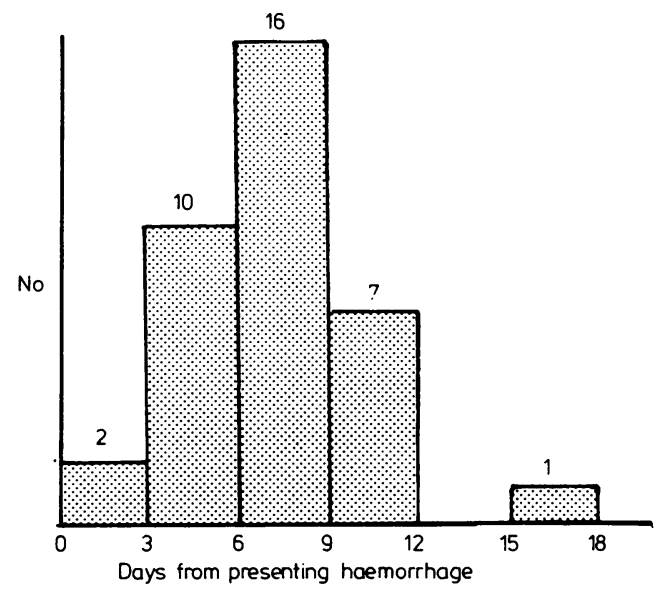

Fig 2 Time course of non-haemorrhagic deterioration.

subsequent rebleeds without having recovered from the episode of deterioration and six were left with fixed neurological deficits. Seventeen made complete recoveries. (4) Possible aetiological factors: Intracerebral haematoma possibly implicated 4, Hydrocephalus possibly implicated 0 , Hemisphere/ lobar swelling possibly implicated 2, onset within 48 hours of angiography 10. (5) Relationship to admission grade, site of aneurysm and age: These are shown in tables 1-3. (6) Relationship to rebleeding: $7 / 36$ patients $(19 \%)$ rebled after having had an episode of deterioration compared with 14/74 (19\%) who rebled without any preceding episode of non-haemorrhagic deterioration.

\section{(c) CEREBRAL VASOSPASM SHOWN ON} ANGIOGRAPHY

Seventy-seven patients had cerebral angiography carried out between days 4-12 from the presenting haemorrhage, coinciding with the period when it is known that cerebral vasospasm is at a maximum after subarachnoid haemorrhage. ${ }^{12}$ Of these patients 15 rebled and 27 had non-haemorrhagic deterioration. Vasospasm was demonstrated to some extent in $80 \%$ (12 patients) of those who rebled and $78 \%$ ( 21 patients) of those who underwent non haemorrhagic deterioration. On the other hand, vasospasm was found in only $29 \%$ (10 patients) of those who had an entirely stable clinical course. The relationship between the incidence of vasospasm and clinical grade on admission was as follows: Grade 1: $45 \%$ (13/29); Grade 2: $55 \%$ (12/22); Grade 3: $58 \%$ (11/19); Grade 4: $100 \%$ (7/7). 


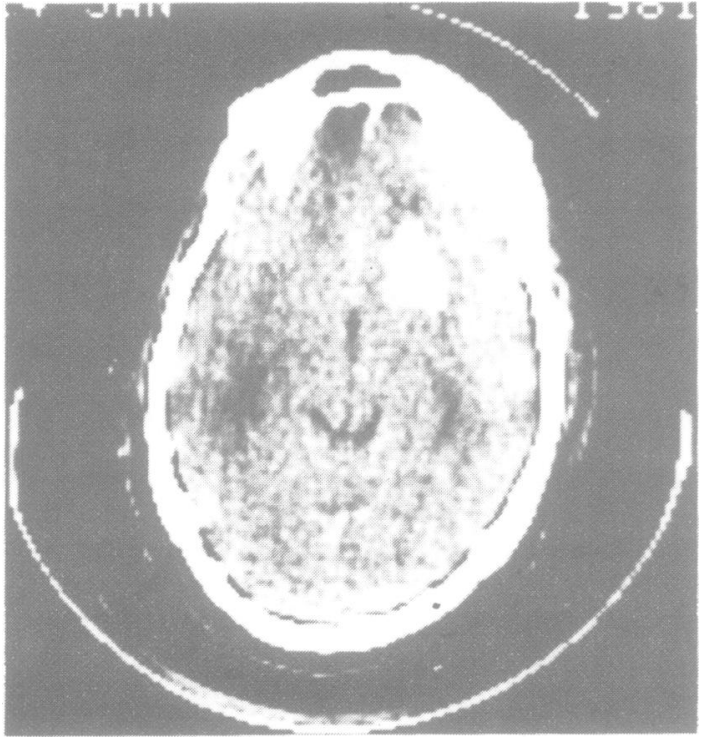

Fig 3 CT scan appearances of NHD and rebleeding in the one patient. (Serial CT scans follow in figs 4, 5): five days after haemorrhage from an aneurysm at the bifurcation of the right internal carotid artery. There is a small haematoma in the basal ganglia.

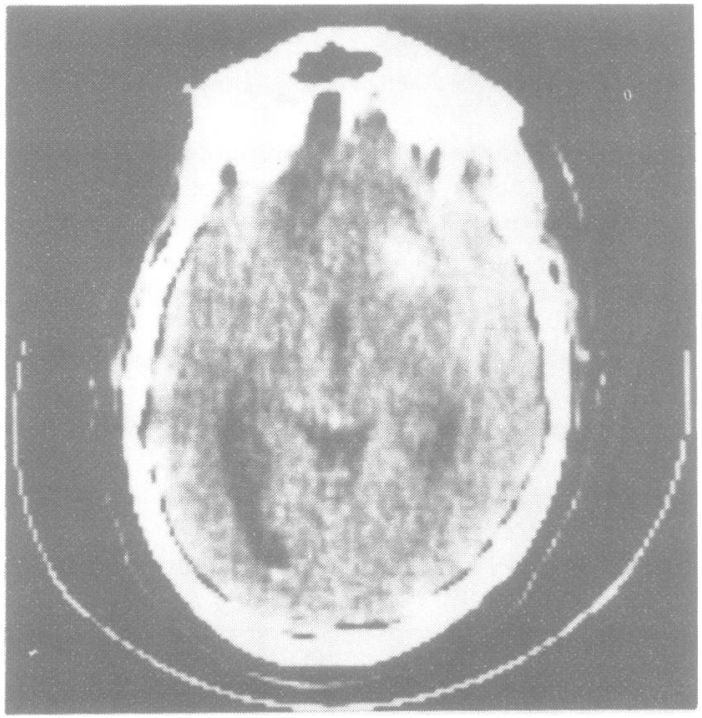

Fig 4 Five days after the previous scan. The patient deteriorated abruptly with increased headache, drowsiness and increased signs of meningeal irritation. Lumbar puncture revealed no fresh blood and the CSF was at a pressure of $120 \mathrm{mms}$. The CT scan shows that the original haematoma is resolving.

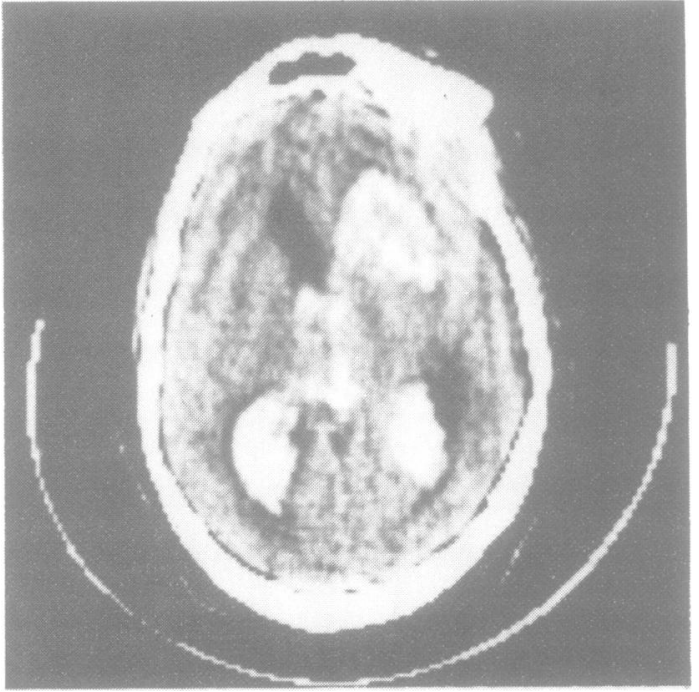

Fig 5 Six days after the second scan. The patient had begun to improve and then once more deteriorated in a similar fashion. A rebleed into the ventricular system is confirmed.

\section{Discussion}

In any series of ruptured aneurysms there is bound to be some distortion of the natural history of the condition caused by selected referral of cases and by early surgical intervention. However in this series, all cases of subarachnoid haemorrhage under the age of 65 years were admitted as soon as possible after referral regardless of their clinical state and only $21 \%$ underwent surgery within 10 days of their presenting haemorrhage.

Four cases had been devastated by the initial bleed and arrived in a moribund and deteriorating state (grade 5). These patients all died within 48 hours of arrival from the effects of the primary haemorrhage. The remaining 106 cases had stabilised or had begun to improve after their bleed. Forty-nine of these subsequently showed marked neurological deterioration, involving 57 episodes of deterioration in all. Confirmed recurrent bleeding accounted for less than a third of those episodes of delayed deterioration which took place within the first three weeks of the presenting haemorrhage.

The other episodes are best designated by the term "non-haemorrhagic deterioration" which does not beg the question of pathogenesis. In a few cases factors such as hydrocephalus or focal intracranial swelling (haematomas or oedema) could be implicated, but in the majority definite aetiology could not be identified. It is widely believed that delayed cerebral vasospasm accounts for many of these 
episodes of non-haemorrhagic deterioration although the degree of correlation with vasospasm as demonstrated on angiography is limited. ${ }^{24}$

There were clinical differences between rebleeding and non-haemorrhagic deterioration. The most striking difference lay in their time courses. The onset of non-haemorrhagic deterioration rose to a peak between days 4-12 and no cases occurred after the 16th day from the last haemorrhage. By contrast, the distribution in time of rebleeding was even during the first three weeks, and rebleeding could occur much later.

Rebleeding had a very high mortality $(90 \%)$ as compared with non-haemorrhagic deterioration (22\%). About half the affected patients made a complete recovery from their episodes of nonhaemorrhagic deterioration. This meant that during the first three weeks the risk of suffering a serious consequence from non-haemorrhagic deterioration was only slightly greater than from rebleeding. During this period, eleven patients died from rebleeding, while non-haemorrhagic deterioration led to eight deaths and left a further six patients with a significant permanent neurological deficit.

The onset of non-haemorrhagic deterioration tended to be gradual whereas the onset of rebleeding was generally abrupt. However, it was seldom possible to determine the mode of onset with absolute confidence, unless the patient was being attended to at the time that deterioration began. It was frequently difficult to distinguish between rebleeding and non-haemorrhagic deterioration on clinical grounds. Patients with non-haemorrhagic deterioration often had increased headache and meningism, while patients with confirmed rebleeds might have none.

A tendency to rebleeding or non-haemorrhagic deterioration did not appear to be related to the age of the patient. Non-haemorrhagic deterioration was slightly commoner $(46 \%)$ in patients with anterior circle aneurysms closely related to the diencephalon (anterior communicating and internal carotid aneurysms) as compared with aneurysms situated elsewhere $(22 \%)$, but the incidence of rebleeding differed little according to the site of the aneurysm. Patients who were more obtunded at the time of admission (grades 3 and 4) were somewhat more liable to both non-haemorrhagic deterioration and rebleeding, but not markedly so, but nonhaemorrhagic deterioration leading to death or a permanent neurological deficit was not more frequent in patients in the higher clinical grades.

These findings are in marked contrast to those of the earlier classic studies of the natural history of ruptured aneurysm which form the basis of currently accepted beliefs about the course of this condition.
These early studies make little or no mention of the fact that delayed deterioration from causes other than rebleeding may be a common event in those patients who arrive at hospital in a good or fair condition. $^{5-10}$ It seems to have been assumed that any worsening in the condition of a previously stable patient must be due to recurrent haemorrhage. Only in the last 10 years has it been largely recognised that a number of conditions may give rise to delayed deterioration in addition to rebleeding. ${ }^{41}$ Of these other causes, cerebral vasospasm has been assumed to be the most common.

Deciding whether an episode of deterioration is caused by rebleeding can be difficult in the first few days after the presenting haemorrhage if repeat lumbar puncture or necropsy are the only tests available. Until the development of the CT scan it was not possible to be certain about the nature of many of these early episodes. ${ }^{11}$

The Multicentre Co-operative Study on intracranial aneurysms found that the daily incidence of rebleeding rose from an initially low level to a sharp peak between the fifth and eleventh day after which it fell away steadily. ${ }^{5}$ The same occurrence was observed by others. $^{8912}$ The explanation which has been given for this phenomenon is that the initial rise is caused by progressive lysis of the fibrin plug which has sealed the hole in the aneurysm and the subsequent decline by the maturation of the definitive fibroblastic repair process. ${ }^{13}$

It will be seen that this peak coincides with the peak of non-haemorrhagic deterioration which has been demonstrated in this study. It also coincides with the period during which vasospasm as demonstrated by angiography, commences and reaches a peak. ${ }^{2}$ It is difficult to believe that rebleeding, non-haemorrhagic deterioration and vasospasm have exactly the same time course. It is easy to see that vasospasm and non-haemorrhagic deterioration may be causally related and thus have the same pattern in time, but one would expect that, if anything, vasospasm would have a protective effect against rebleeding, so that if a "peak" of rebleeding did occur, it would be after the second week as the vasospasm eventually wore off.

There are three possible explanations for the temporal coincidence of these three phenomena. The first is that the earlier studies failed to distinguish between non-haemorrhagic deterioration and early rebleeding. Even in the Co-operative Study, only about $60 \%$ of all rebleeds, both early and late, appear to have been confirmed by lumbar puncture or necropsy. ${ }^{5}$ In the present study every episode of neurological deterioration was carefully and critically assessed at the time that it occurred. Every effort was made to establish that an episode 
was due to recurrent haemorrhage. Serial CT scans and lumbar punctures were carried out as necessary. Even so, no evidence of rebleeding could be found in two-thirds of the early episodes of deterioration. The confirmed rebleeds did not show any "peaking" in their time pattern.

This explanation is supported by two other pieces of evidence. First, the present study shows a very high mortality of rebleeds $(90 \%)$ as compared with the mortality of rebleeding reported by early papers $(40-60 \%)^{591214}$ but the mortality of the cases of non-haemorrhagic deterioration and rebleeding taken together $(55 \%)$ approximates to the level of the latter. Second, the total number of episodes of rebleeding added together in the first three weeks (29) is close to the number of rebleeds (27) that the Co-operative Study would have predicted during this period. ${ }^{5}$

The second possible explanation is that episodes of non-haemorrhagic deterioration are really micro-bleeds, too small to be detected by repeat lumbar puncture or CT scans. However, patients who had an episode of non-haemorrhagic deterioration were not more liable to later frank rebleeding that those who had had a more stable course. Furthermore, patients whose aneurysms were explored after an episode of non-haemorrhagic deterioration had occurred, did not appear to have evidence of any bleeding which was more recent than the presenting haemorrhage.

The third possible explanation is that in this series the natural history of the condition had been grossly modified by the administration of the antifibrinolytic drug, tranexamic acid. If this is so, then the effect of the drug would be to replace one problem (rebleeding) with another (non-haemorrhagic deterioration), the characteristic time course of the latter replacing that of the former which has become more flattened and uniform. This would seem a surprising effect. Several controlled trials have shown an apparent reduction in rebleeding when antifibrinolytic drugs are used ${ }^{15-19}$ but other trials have failed to do so. ${ }^{19-22}$ Some of these trials have been open and perhaps an open trial permits some observer bias with regard to the question of whether an episode of deterioration is a rebleed or not.

The findings of this study have two implications. First, they should prompt re-examination of the possible efficacy and effects of antifibrinolytic therapy. There is a need for a large-scale multicentre double-blind controlled trial. Second, whether or not antifibrinolytic drugs really do affect the natural history of ruptured aneurysms, it now seems that rebleeding is much less common than non-haemorrhagic deterioration in the three weeks after the first rupture. Because of the high mortality and morbidity which accompany early surgery, many surgeons now postpone surgery to at least $\mathbf{1 0}$ days from the last bleed, even if the patient is in good condition. ${ }^{23} 24$ It may be that the risks of the patient dying from rebleeding during this waiting period are much lower than is generally believed and that such a waiting policy can thus be advocated with more confidence. During the course of the present study only three patients admitted in Botterell grades 1-2 died from rebleeding within the first 10 days and in two of these cases the rebleeding occurred so soon after arrival in the neurosurgical unit that surgery would not have been carried out even if a deliberate policy of early operation had been followed.

Perhaps one should consider whether angiography should normally be delayed until the end of the waiting period. In 10 cases in this series, nonhaemorrhagic deterioration began within 48 hours of angiography. In selected patients (for example those with haematomas behaving as mass lesions) early angiography might be indicated, but otherwise, assuming that many instances of non-haemorrhagic deterioration are related to vasospasm, it would seem irrational to perform angiography during the period when vasospasm is known to be at its maximum if surgery is not going to be carried out straight away.

\section{References}

' Weir B, Grace M, Hansen J, Rothberg C. The course of vasospasm in man. $J$ Neurosurg 1978;48:173-8.

${ }^{2}$ Saito I, Ueda Y, Sano K. Significance of vasospasm in the treatment of ruptured intracranial aneurysms. $J$ Neurosurg 1977;47:412-29.

${ }^{3}$ Hunt WE, Hess RM. Surgical risk as related to time of intervention in the repair of intracranial aneurysms. $J$ Neurosurg 1968;28:14-19.

${ }^{4}$ Mohan J. In: Boullin DJ, ed. Cerebral Vasospasm. Chichester: John Wiley, 1980:15-35.

${ }^{5}$ Locksley HB. Natural history of subarachnoid hemorrhage, intracranial aneurysms and arteriovenous malformations. J Neurosurg 1966;25:321-68.

${ }^{6}$ Hamilton JG, Falconer MA. Immediate and late results of surgery in cases of saccular intracranial aneurysms. $J$ Neurosurg 1959;16:514-41.

7 Walton JN. The late prognosis of subarachnoid haemorrhage. Br Med J 1952;2:802-3.

${ }^{8}$ Magee CG. Spontaneous subarachnoid haemorrhage: a review of 150 cases. Lancet 1943;2:497-500.

${ }^{9}$ McKissock W, Walsh L. Subarachnoid haemorrhage due to intracranial aneurysms. Br Med J 1956;2:559-65.

${ }^{10}$ Logue V. Surgery in spontaneous subarachnoid haemorrhage. Br Med J 1956;1:473-9.

1 Van Crevel H. Pitfalls in the diagnosis of rebleeding from intracranial aneurysm. Clin Neurol Neurosurg 1980;82:1-9. 
12 Pakarinen A. Incidence, aetiology and prognosis of primary subarachnoid haemorrhage. Acta Neurol Scand 1967;43 Suppl 29:9-128.

${ }^{13}$ Maurice-Williams RS, Gordon YB, Sykes A. Monitoring fibrinolytic activity in the cerebrospinal fluid after subarachnoid haemorrhage: a guide to the risk of rebleeding? J Neurol Neurosurg Psychiatry 1980; 43:175-81.

14 Trumpy JH. Subarachnoid haemorrhage. Time sequence of recurrences and their prognosis. Acta Neurol Scand 1967;43:48-60.

${ }^{15}$ Maurice-Williams RS. Prolonged antifibrinolysis: an effective non-surgical treatment for ruptured intracranial aneurysms? Br Med J 1978;1:945-7.

${ }^{16}$ Chandra B. Treatment of subarachnoid haemorrhage from ruptured intracranial aneurysms with tranexamic acid: a double blind clinical trial. Ann Neurol 1978;3:502-4.

${ }^{17}$ Chowdhary UM, Carey PC, Hussein MM. Prevention of early recurrence of spontaneous subarachnoid haemorrhage by $\epsilon$-aminocaproic acid. Lancet 1979;1:741-3.

${ }^{18}$ Sengupta RP, So SC, Villarejo-Ortega FJ. Use of epsilon-aminocaproic acid (EACA) in the pre- operative management of ruptured intracranial aneurysms. J Neurosurg 1976;44:476-84.

${ }^{19}$ Fodstad H. Tranexamic acid as a therapeutic agent in aneurysmal subarachnoid haemorrhage: clinical, laboratory and experimental studies. Umea University Medical Dissertations New Series No 60, 1980.

${ }^{20}$ Rossum J van, Wintzen AR, Endtz LJ, Schoen JHR, Jonge $\mathrm{H}$. Effect of tranexamic acid on rebleeding after subarachnoid haemorrhage. Ann Neurol 1977;2: 238-42.

${ }^{21}$ Shucart WA, Hussain SK, Cooper PR. Epsilonaminocaproic acid and recurrent subarachnoid hemorrhage. J Neurosurg 1980;53:28-31.

${ }^{22}$ Kaste K, Ramsay M. Tranexamic acid in subarachnoid haemorrhage. A double-blind study. Stroke 1979; 10:519-21.

${ }^{23}$ Adams CBT, Fearnside MR, O'Laoire SA. Intracranial aneurysm: analysis of results of microneurosurgery in 100 consecutive patients. $\mathrm{Br}$ Med J 1976;2:607-9.

${ }^{24}$ Mullan S. The initial medical management of ruptured intracranial aneurysms. In: Current controversies in Neurosurgery. Philadelphia: WB Saunders Co., 1976:259-69. 